\title{
Treatment of a glioblastoma patient by vaccination with autologous dendritic cells pulsed with allogeneic major histocompatibility complex class I-matched tumor peptides
}

\author{
Case report
}

\author{
Linda M. Liau, M.D., Ph.D., Keith L. Black, M.D., Neil A. Martin, M.D., \\ Steven N. Sykes, B.S., Jeff M. Bronstein, M.D., Ph.D., LiSa Jouben-Steele, M.D., \\ Paul S. Mischel, M.D., Arie Belldegrun, M.D., and Timothy F. Cloughesy, M.D. \\ Division of Neurosurgery, and Departments of Neurology, Pathology, and Urology, University of \\ California at Los Angeles School of Medicine; and the Maxine Dunitz. Neurosurgical Institute, \\ Cedars-Sinai Medical Center, Los Angeles, California
}

\begin{abstract}
Dendritic cells (DCs) are antigen-presenting cells that play a central role in the initiation and modulation of antitumor immune responses. In this pilot study, we investigated the ability of autologous DCs pulsed ex vivo with allogeneic major histocompatibility complex class I-matched glioblastoma peptides to stimulate host antitumor immune responses when injected as a vaccine.

A patient with recurrent brainstem glioblastoma multiforme (GBM) received a series of three intradermal immunizations of antigen-pulsed DCs on an outpatient basis following surgical debulking of her posterior fossa tumor. Dendritic cell vaccination was well tolerated, and no clinical signs of autoimmunity or experimental allergic encephalomyelitis were detected. She developed a measurable cellular immune response against the allogeneic glioblastoma peptides used in her vaccine preparation, as demonstrated by in vitro T-cell proliferation assays. In addition, increased T-cell infiltration was noted within the intracranial tumor site in the biopsy sample obtained following DC vaccination. An objective clinical response, however, was not evident, and this patient eventually died 21 months after her disease was diagnosed.

To our knowledge, this is the first patient with brain cancer ever to be treated with DC-based immunotherapy. This case illustrates that vaccination with DCs pulsed with acid-eluted glioblastoma peptides is feasible and can induce systemic antigen-specific immunity in a patient with recurrent GBM. Additional studies are necessary to determine the optimum DC doses and antigen loading conditions that may translate into clinical effectiveness and survival benefit for patients with brain tumors. Phase I trials for malignant glioma are currently underway.
\end{abstract}

\section{KEY WORDS • dendritic cell • immunotherapy • brain tumor • tumor antigen • glioblastoma multiforme • major histocompatability complex}

As surgery, chemotherapy, and radiotherapy have failed to improve the poor prognosis of patients with GBM, ${ }^{6}$ there has been increased motivation to develop novel, alternative therapies for these deadly neoplasms..$^{21}$ One such alternative is to utilize the immune system to specifically target and eliminate tumor cells on the basis of antigen expression and recognition. ${ }^{20}$ It is now well established that tumor cells express certain proteins that are different from nontumor cells ${ }^{12,24}$ and, additionally, that the immune system has cells, particularly cytotoxic $\mathrm{T}$ lymphocytes, that can recognize tumor antigens and kill tumors. ${ }^{25,32}$ However, a major problem is that antitumor immunity is

Abbreviations used in this paper: $\mathrm{CNS}=$ central nervous system; $\mathrm{DC}=$ dendritic cell; GBM = glioblastoma multiforme; $\mathrm{MHC}=$ major histocompatibility complex; $\mathrm{MR}=$ magnetic resonance; UCLA = University of California at Los Angeles. not effectively induced in tumor-bearing hosts. Although tumors have antigens for T cells, these do not appear to be immunogenic in vivo. ${ }^{19}$ One possible reason is that there is insufficient tumor antigen presentation to elicit T-cell immunity. ${ }^{26,27}$ This is particularly true for brain tumors, as $\mathrm{T}$ cells are not readily evident within the immune-privileged CNS unless T-cell immunity is generated.

Dendritic cells are antigen-presenting cells specialized for the induction of primary T-cell responses. ${ }^{2}$ They possess the necessary costimulatory molecules and cytokine profiles for eliciting effective T-cell immunity. These cells capture and convert protein antigens into peptide fragments, load them onto MHC molecules on their cell surfaces, and then present the peptide-MHC complexes to T lymphocytes for stimulating antigen-specific T-cell immune responses. ${ }^{13,31}$ Dendritic cells play a central role in the initiation of antitumor immune responses and are con- 


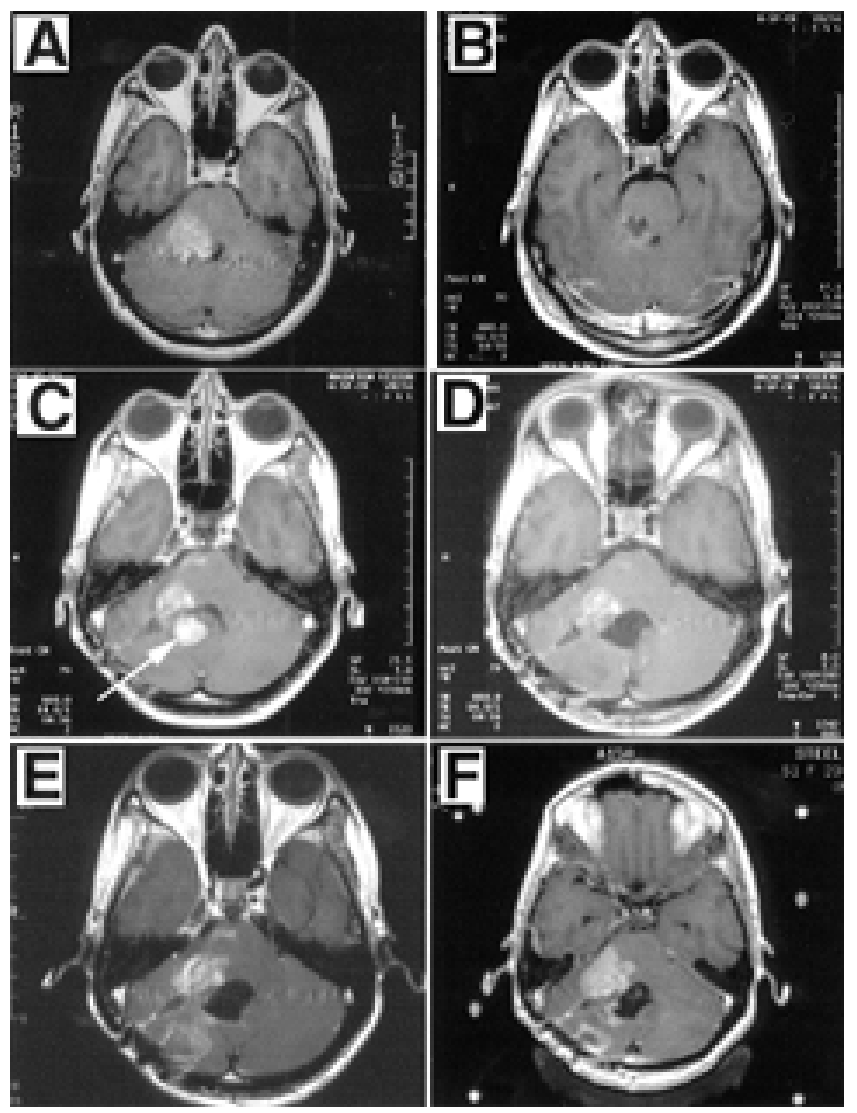

Fig. 1. Axial gadolinium-enhanced $\mathrm{T}_{1}$-weighted $\mathrm{MR}$ images. A: Image obtained at initial tumor diagnosis. B: Postoperative image obtained following first resection in April 1996. C: Eleven months later, imaging revealed tumor recurrence at the previous resection site and in a new area in the right cerebellar tonsil and vermis, just posterior to the original lesion (arrow). D: Postoperative image following surgical debulking of recurrent tumor and just before three biweekly vaccinations of allogeneic tumor peptide-pulsed DCs (April 1997). Note the residual bulk tumor prior to immunotherapy. E: Image obtained 2 weeks after the cycle of DC vaccinations, revealing no evidence of clinical response following treatment trial. F: Two months after completion of the vaccine treatments, imaging demonstrated evidence of continued tumor progression into the pontocerebellar junction. The patient died 6 months later.

sidered to be promising adjuvants for inducing immune responses to cancer. ${ }^{29}$ Initial data obtained from clinical trials of DC-based vaccines for B-cell lymphoma, ${ }^{9}$ melanoma, ${ }^{17,29}$ prostate cancer, ${ }^{15,16,30}$ and renal cell carcino$\mathrm{ma}^{8,10}$ have recently been published, and the results appear to be encouraging. In different animal models, the capacity of DCs to elicit antitumor immune responses for the treatment of CNS tumors has been demonstrated by our laboratory and others. ${ }^{1,7,11,18}$

As a feasibility study and preliminary proof of the concept for clinical trials in patients with brain tumors, we report on the results of the first patient with GBM to be treated with a full course of peptide-pulsed DC immunotherapy. This single-patient trial was conducted at UCLA Medical Center in April of 1997 and, to our knowledge, represents the first human test of DC immunotherapy for brain cancer.

\section{CASE REPORT}

History and Prior Therapy. This 49-year-old woman presented to UCLA Medical Center in March 1996 with a several-week history of progressive right-sided facial numbness, diplopia, and gait ataxia. Brain MR imaging revealed an enhancing lesion of the right middle cerebellar peduncle and right lateral pons, suggestive of a tumor (Fig. 1A). She underwent resection of this lesion in April 1996 (Fig. 1B), and pathological examination confirmed a diagnosis of GBM. She then underwent hyperfractionated radiotherapy (5400 cGy) and received Accutane and highdose Tamoxifen therapy. Eleven months after her initial diagnosis, follow-up MR imaging demonstrated tumor recurrence in a new site in the right cerebellar tonsil and vermis, just posterior to the original lesion (Fig. 1C). In March 1997 the recurrent tumor was surgically debulked, and postoperative MR imaging revealed subtotal resection of the tumor in the region of the right cerebellar tonsil and some residual enhancement (Fig. 1D). Because previous standard treatment regimens had failed, a compassionate waiver was obtained to treat this patient with peptidepulse DC immunotherapy in a single-patient study protocol approved by the UCLA institutional review board.

Dendritic Cell Immunotherapy. Two weeks following her second surgery, leukocytes were prepared from $100 \mathrm{ml}$ of the patient's peripheral blood by using Ficoll-Hypaque density centrifugation. Dendritic cells were generated as described previously. ${ }^{3,23}$ Briefly, peripheral blood monocytes were resuspended in clinical-grade RPMI 1640 plus $10 \%$ autologous human serum and allowed to adhere to plastic culture dishes. After 2 hours at $37^{\circ} \mathrm{C}$, the nonadherent cells were removed, and the adherent cells were cultured for 7 days with GM-CSF (2000 U/ml) and IL-4 (1000 U/ml). Phenotypic changes were monitored by light microscopy; FACScan flow cytometry analysis revealed the cells to express high levels of MHC class I, MHC class II, and costimulatory molecules $\mathrm{CD} 80^{+}$(B7.1) and CD86+ (B7.2) (data not shown).

Tumor peptides were eluted from primary cultures of an allogeneic, MHC class I-matched glioblastoma, according to previously published protocols. ${ }^{10,33}$ Briefly, $4 \times 10^{9}$ of tumor cells obtained from a viable primary glioblastoma cell culture were washed three times with sterile phosphate-buffered saline and collected by centrifugation. The cell pellet was gently triturated for 30 seconds in $5 \mathrm{ml}$ citrate-phosphate buffer ( $\mathrm{pH} 3.1)$, which has been shown to effectively elute tumor surface peptides bound to MHC class I molecules. ${ }^{28}$ Peptides derived from the cell-free supernatant were then purified on an activated SepPak 18 column, eluted with $1.5 \mathrm{ml} 60 \%$ acetonitrile in water, lyophilized to near complete dryness $(50 \mu \mathrm{l})$, and stored at $-20^{\circ} \mathrm{C}$.

In early April 1997, the patient underwent the first of three biweekly immunizations with glioblastoma peptidepulsed DCs. For each injection, $5 \times 10^{6}$ of the patient's autologous DCs were cocultured overnight (16-24 hours) with $160 \mu \mathrm{g}$ of eluted tumor peptide. On the days of immunization, the peptide-pulsed DCs were washed, resuspended in $1 \mathrm{ml}$ sterile phosphate-buffered saline in a $25-$ gauge tuberculin syringe, and injected intradermally into the left-sided axilla.

The treatments were well tolerated, and no significant 


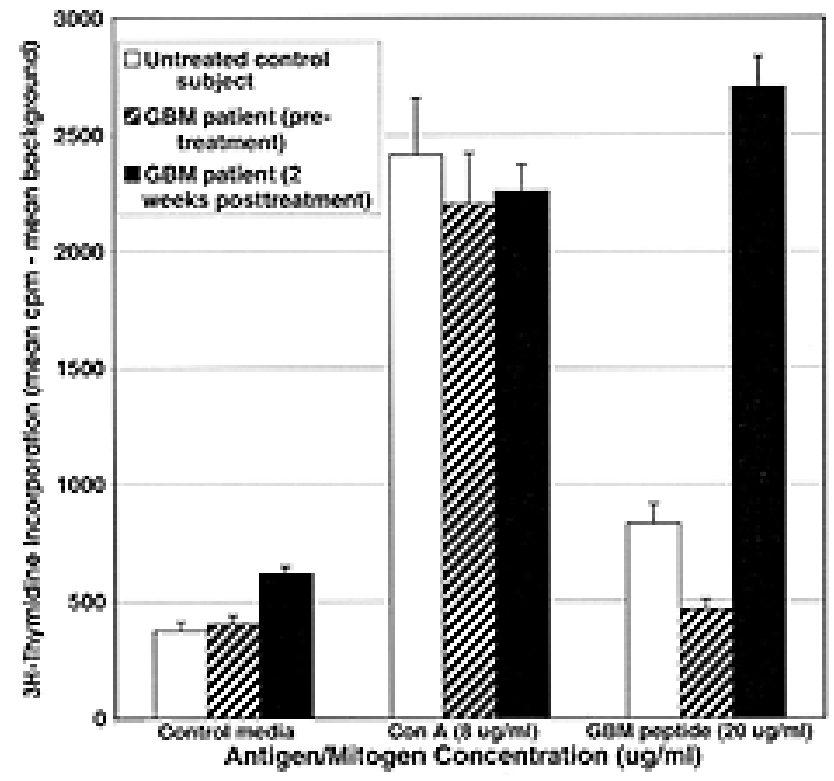

Fig. 2. Bar graph showing the peripheral blood mononuclear cell (PBMC) T-cell proliferation response. The PBMCs were cocultured in vitro with control serum-free media, media containing mitogen Con A $(8 \mu \mathrm{g} / \mathrm{ml})$, or media with the allogeneic acid-eluted tumor peptides $(20 \mu \mathrm{g} / \mathrm{ml})$ used to prepare the DC vaccine. Incorporation of $\left[{ }^{3} \mathrm{H}\right]$ thymidine is reported as mean counts per minute $(\mathrm{cpm})$ of quadruplicate wells above the media background. Error bars represent standard deviations. The PBMCs were obtained from an untreated control subject and from the patient pretreatment and 2 weeks following her last injection of DCs. In control media, no significant proliferative responses were seen in any of the samples. Conversely, in response to the universal mitogen Con $\mathrm{A}$, robust $\left[{ }^{3} \mathrm{H}\right]$ thymidine incorporation was seen in all the samples, which confirms that the PBMCs used in this assay could respond to the appropriate proliferative stimuli. In response to the GBM tumor peptides, incorporation of $\left[{ }^{3} \mathrm{H}\right]$ thymidine was greater than 2,500 counts/minute 2 weeks after the DC injections in our patient. This antigen-specific T-cell proliferation in response to tumor peptide was markedly higher than that seen pretreatment or in the control subject.

side effects were associated with the actual injection of peptide-pulsed DCs. However, by the end of the 3rd week of the trial, the patient was developing increased ataxia and worsening diplopia, suggestive of tumor progression in her brainstem. A follow-up MR imaging scan revealed enlargement of the residual tumor as well as edema and compression of the fourth ventricle. The patient therefore underwent a debulking procedure for decompression of the brainstem in late April 1997. Two weeks later, the patient completed the last of her planned three DC treatments, which she tolerated well.

Immunological Response. Blood samples were collected before the fist DC treatment and two weeks after the last immunization. Following treatment, the patient developed a strong T-cell proliferative response against the allogeneic tumor peptide preparation that was used to pulse her DCs for vaccination (Fig. 2). This response was specific in our patient for the injected allogeneic glioblastoma peptide, as it was not seen pretreatment. Furthermore, unlike the T cells obtained from our patient, peripheral blood mononuclear cells collected from an untreated control

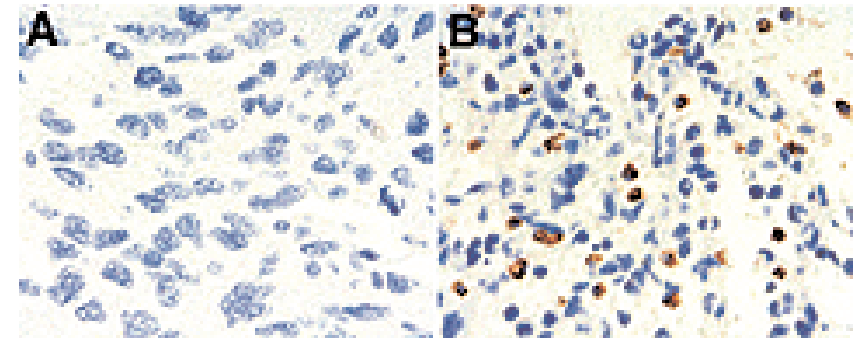

Fig. 3. Immunohistochemical analysis of immune infiltrates into brain tumor specimens. A: Analysis of a biopsy specimen of GBM taken before vaccination with peptide-pulsed DCs revealed no evidence of T-cell infiltration. B: Following DC immunizations, analysis of the tumor specimens demonstrated an increased infiltration of $\mathrm{CD}^{+} \mathrm{T}$ lymphocytes among glioblastoma cells. Immunoperoxidase staining with a CD3 monoclonal antibody with hemotoxylin counterstain, original magnification $\times 400$.

subject did not show a significant proliferative response to the glioblastoma tumor peptide.

Because this patient underwent surgical tumor debulking after having received two vaccinations of peptidepulsed DCs, we were able to obtain postvaccination brain tumor specimens for evaluation of possible immunological responses within the CNS. Staining of cryosections with anti-CD3 monoclonal antibody revealed increased $\mathrm{CD}^{+} \mathrm{T}$-cell inflammatory infiltrates into the tumor (Fig. 3 ). There was no evidence of experimental allergic encephalomyelitis, hemorrhage, or excessive necrosis.

Clinical Response. Despite having a significantly measurable T-cell proliferative response against the tumor peptide used for the peptide-pulsed DC treatment, the patient developed progressive disease 2 months after the series of DC vaccinations. Follow-up brain MR imaging revealed increased tumor mass in the right-sided middle cerebellar peduncle, extending into the pontocerebellar junction (Fig. 1F). The patient underwent stereotactic fractionated radiosurgery in June of 1997 and treatment with antineoplastins in October of 1997. Despite these therapies, the patient suffered progressive tumor growth and eventually died of her disease in December 1997.

\section{Sources of Supplies and Equipment}

The RPMI 1640 was purchased from Life Technologies (Gaithersburg, MD). The culture dishes were acquired from Costar (Cambridge, MA). Immunex (Seattle, WA) manufactures the GM-CSF. We obtained the SepPak 18 column from Millipore (Bedford, MA) and the IL-4 from Shering-Plough Research (Kenilworth, NJ).

\section{DISCUSSION}

This is the first case report of tumor antigen-pulsed DC therapy in a patient with GBM. In this preliminary study, we show that DC-based vaccination in patients glioblastoma is feasible and can induce potentially relevant immunological responses. Our results indicate that DCs can be safely harvested in patients with such lesions, pulsed with acid-eluted tumor peptides from primary glioma cell cultures, and injected repeatedly into the patient without causing significant toxicity. Sufficient numbers of DCs 
were generated from $100 \mathrm{ml}$ blood samples without the need for leukapheresis or priming with hematopoietic growth factors. In this case vaccination with peptidepulsed DCs was a well-tolerated outpatient treatment, and no clinical signs of experimental allergic encephalomyelitis were observed.

Using peripheral blood mononuclear cells taken from this patient for in vitro proliferation assays as a method of detecting antigen-specific immunity, a significant tumor peptide-specific T-cell proliferative response developed against the tumor peptides that were used to pulse the DC vaccine. This response occurred as early as 2 weeks after the patient's last immunization with DCs and was induced with as few as five million DCs per injection, attesting to the potency of this form of treatment for inducing cellular immune responses. In addition, examination of biopsy specimens taken from the intracranial tumor site revealed increased infiltration of $\mathrm{CD}^{+} \mathrm{T}$ lymphocytes following DC vaccination, suggesting possible propagation of antitumor T-cell immune responses within the CNS.

Despite these promising immunological responses, however, an objective clinical response was not observed in this single-patient trial. In a pilot study such as this, the assessment of clinical response was not our primary aim, as no definitive conclusions regarding possible clinical benefits could realistically be made. Nevertheless, several reasons may be postulated as to why a significant beneficial effect was not observed in this patient. First, our patient had a Karnofsky performance scale index of $70 \%$, and all previous treatment regimens had failed. The relatively low functional performance status and prior treatment failures are predisposing indicators of poor prognosis regardless of the subsequent therapy. Second, given the location of this patient's tumor involving the brainstem, a gross-total resection could not be safely achieved prior to DC vaccination. Therefore, her intracranial tumor load was still relatively high before this adjuvant treatment. It has been proposed that immunotherapy is not best suited for bulk disease and is most effective when the tumor burden is low. ${ }^{17}$

Furthermore, because we were limited by the size of the surgical specimen, we were unable to generate sufficient primary glioblastoma cultures from which to elute autologous tumor peptides for this patient. Although the use of acid-eluted tumor peptides derived from primary cells cultures eliminates the requirement to identify brain tumorspecific or -associated antigens, the ability to generate sufficient autologous primary tumor cultures from surgically resected glioblastoma specimens is not always possible, especially from previously irradiated and necrotic tissues. We therefore used MHC class I-matched tumor peptides eluted from another primary glioblastoma culture for pulsing DCs. Although in vitro T-cell proliferation assays revealed that our patient had developed substantial cellular immune responses against the allogeneic tumor peptides, it is conceivable that her autologous tumor did not share enough tumor antigen homology with the allogeneic donor to induce a strong enough tumor-specific immunity that could eradicate her tumor in vivo. This suggests that a vaccination approach in which DCs are cocultured with allogeneic MHC class I-matched tumor peptides may be of limited potency and that autologous sources of antigen may be more effective. Nevertheless, reliance on autologous tumor antigens derived from small surgical glioma specimens may be a practical limitation. Some approaches that may overcome this drawback are the use of synthetic tumor-associated antigen peptides ${ }^{16}$, ${ }^{18,29}$ or amplified tumor messenger $\mathrm{RNA}^{1,4}$ as sources of antigen for DCs. These and other alternative strategies of antigen pulsing of DCs are actively being investigated in preclinical studies. ${ }^{5}$

In conclusion, the preliminary results from this case study provided us with important lessons for the design of subsequent Phase I clinical trials of DC-based immunotherapy for malignant gliomas. The data indicated that in a patient with GBM, vaccination with peptide-pulsed DCs is feasible, well tolerated, and induces promising immunological responses. Additional preclinical and clinical studies are necessary to elucidate how these immunological endpoints can be effectively translated into clinical benefits for patients with CNS tumors. Future experimental protocols will focus on determining the optimum dose of DCs, the appropriate frequency and route of vaccinations, and the best source of tumor antigens, as well as alternative methods of antigen loading. ${ }^{14,22}$ Optimization of these parameters will be necessary to address the true clinical effectiveness of this immunotherapeutic approach for brain cancer.

\section{References}

1. Ashley D, Faiola B, Nair S, et al: Bone marrow-generated dendritic cells pulsed with tumor extracts or tumor RNA induce antitumor immunity against central nervous system tumors. J Exp Med 186:1177-1182, 1997

2. Banchereau J, Steinman RM: Dendritic cells and the control of immunity. Nature 392:245-252, 1998

3. Bender A, Sapp M, Schuler G, et al: Improved methods for the generation of dendritic cells from nonproliferating progenitors in human blood. J Immunol Methods 196:121-135, 1996

4. Boczkowski D, Nair S, Snyder D, et al: Dendritic cells pulsed with RNA are potent antigen-presenting cells in vitro and in vivo. J Exp Med 184:465-472, 1996

5. Broder H, Anderson A, Odesa SK, et al: Recombinant adenovirus-transduced dendritic cell immunization in a murine model of central nervous system tumor. Neurosurg Focus 9 (6):Article 6, 2000

6. Davis FG, Freels S, Grutsch J, et al: Survival rates in patients with primary malignant brain tumors stratified by patient age and tumor histological type: an analysis based on Surveillance, Epidemiology, and End Results (SEER) data, 1973-1991. J Neurosurg 88:1-10, 1998

7. Heimberger AB, Crotty LE, Archer GE, et al: Bone marrowderived dendritic cells pulsed with tumor homogenate induce immunity against syngeneic intracerebral glioma. J Neuroimmunol 103:16-25, 2000

8. Holtl L, Rieser C, Papesh C, et al: Cellular and humoral immune responses in patients with metastatic renal cell carcinoma after vaccination with antigen pulsed dendritic cells. J Urol 161:777-782, 1999

9. Hsu F, Benike C, Fagnoni F, et al: Vaccination of patients with B-cell lymphoma using autologous antigen-pulsed dendritic cells. Nat Med 2:52-58, 1996

10. Kugler A, Stuhler G, Walden P, et al: Regression of human metastatic renal cell carcinoma after vaccination with tumor celldendritic cell hybrids. Nat Med 6:332-336, 2000

11. Liau LM, Black KL, Prins RM, et al: Treatment of intracranial gliomas with bone marrow-derived dendritic cells pulsed with tumor antigens. J Neurosurg 90:1115-1124, 1999 


\section{Dendritic cell-based immunotherapy for glioblastoma}

12. Liau LM, Lallone RL, Seitz RS, et al: Identification of a gliomaassociated growth factor gene, granulin, using "differential immuno-absorption." Cancer Res 60:1353-1360, 2000

13. Mellman I, Turley SJ, Steinman RM: Antigen processing for amateurs and professionals. Trends Cell Biol 8:231-237, 1998

14. Morse M, Coleman R, Akabani G, et al: Migration of human dendritic cells after injection in patients with metastatic malignancies. Cancer Res 59:56-58, 1999

15. Murphy G, Tjoa B, Ragde H, et al: Phase I clinical trial: T-cell therapy for prostate cancer using autologous dendritic cells pulsed with HLA-A0201-specific peptides from prostate-specific membrane antigen. Prostate 29:371-380, 1996

16. Murphy G, Tjoa B, Simmons S, et al: Infusion of dendritic cells pulsed with HLA-A2-specific prostate-specific membrane antigen peptides: a phase II prostate cancer vaccine trial involving patients with hormone-refractory metastatic disease. Prostate 38:73-78, 1999

17. Nestle FO, Alijagic S, Gilliet M, et al: Vaccination of melanoma patients with peptide- or tumor lysate-pulsed dendritic cells. Nat Med 4:328-332, 1998

18. Okada H, Tahara H, Shurin M, et al: Bone marrow-derived dendritic cells pulsed with a tumor-specific peptide elicit effective anti-tumor immunity against intracranial neoplasm. Int J Cancer 78:196-201, 1998

19. Paglia P, Guzman CA: Keeping the immune system alerted against cancer. Cancer Immunol Immunother 46:88-92, 1998

20. Pardoll DM: Cancer vaccines. Nat Med 4:525-531, 1998

21. Parney IF, Hao C, Petruk KC: Glioma immunology and immunotherapy. Neurosurgery 46:778-792, 2000

22. Ribas A, Butterfield $\mathrm{LH}, \mathrm{Hu} \mathrm{B}$, et al: Immune deviation and Fas-mediated deletion limit antitumor activity after multiple dendritic cell vaccinations in mice. Cancer Res 60:2218-2224, 2000

23. Romani N, Reider D, Heuer M, et al: Generation of mature dendritic cells from human blood: an improved method with special regard to clinical applicability. J Immunol Methods 196: 137-151, 1996

24. Rosenberg SA, Kawakami Y, Robbins PF, et al: Identification of the genes encoding cancer antigens: implications for cancer immunotherapy. Adv Cancer Res 70:145-177, 1996
25. Schreiber H: Tumor immunology, in Paul WE (ed): Fundamental Immunology. Philadelphia: Lippincott-Raven, 1999, pp 1237-1270

26. Schuler G, Steinman RM: Dendritic cells as adjuvants for immune-mediated resistance to tumors. J Exp Med 186: 1183-1187, 1997

27. Steinman RM: The dendritic cell system and its role in immunogenicity. Ann Rev Immunol 9:271-296, 1991

28. Storkus WJ, Zeh HJI, Salter RD, et al: Identification of T-cell epitopes: rapid isolation of class I-presented peptides from viable cells by mild acid elution. J Immunother 14:94-103, 1993

29. Thurner B, Haendle I, Roder C, et al: Vaccination with Mage3A1 peptide-pulsed mature, monocyte-derived dendritic cells expands specific cytotoxic $\mathrm{T}$ cells and induces regression of some metastases in advanced stage IV melanoma. J Exp Med 190:1669-1678, 1999

30. Tjoa B, Simmons SJ, Bowes VA, et al: Evaluation of Phase I/II clinical trials in prostate cancer with dendritic cells and PSMA peptides. Prostate 36:39-44, 1998

31. Turley SJ, Inaba K, Garrett WS, et al: Transport of peptide$\mathrm{MCH}$ class II complexes in developing dendritic cells. Science 288:522-527, 2000

32. Van den Eynde BJ, van der Bruggen P: T cell defined tumor antigens. Curr Opin Immunol 9:684-693, 1997

33. Zitvogel L, Mayordomo JI, Tjandrawan T, et al: Therapy of murine tumors with tumor peptide-pulsed dendritic cells: dependence on T cells, B7 costimulation, and Thelper cell 1-associated cytokines. J Exp Med 183:87-97, 1996

Manuscript received October 17, 2000.

Accepted in final form November 20, 2000.

This study was supported in part by a National Brain Tumor Foundation grant awarded to Dr. Liau and by the Steel-Roven Family Fund.

Address reprint requests to: Linda M. Liau, M.D., Ph.D., Division of Neurosurgery, University of California at Los Angeles School of Medicine, 10833 Le Conte Avenue, CHS 74-134, Los Angeles, California 90095-6901. email: 1liau@mednet.ucla.edu. 